\title{
The near-IR properties and continuum shapes of high redshift quasars from the Sloan Digital Sky Survey
}

\author{
L. Pentericci ${ }^{1}$, H.-W. Rix ${ }^{1}$, F. Prada ${ }^{2}$, X. Fan ${ }^{3}$, M. A. Strauss ${ }^{4}$, D. P. Schneider ${ }^{5}$, E. K. Grebel ${ }^{1}$, \\ D. Harbeck ${ }^{1}$, J. Brinkmann ${ }^{6}$, and V. K. Narayanan ${ }^{4}$
}

\author{
1 Max-Planck-Institut fur Astronomie, Konigstuhl 17, 69117, Heidelberg, Germany \\ 2 Instituto de Astrofisica de Canarias, 38205 La Laguna, Tenerife, Spain \\ 3 Steward Observatory, The University of Arizona 933 N. Cherry Ave, Tucson, AZ 85721-0065 Arizona, USA \\ ${ }^{4}$ Princeton University Observatory, Princeton 08544, USA \\ ${ }^{5}$ Department of Astronomy and Astrophysics, The Pennsylvania State University, University Park, PA 16802, USA \\ ${ }^{6}$ Apache Point Observatory PO Box 59, Sunspot, NM 88349-0059, USA
}

Received 22 January 2003 / Accepted 4 July 2003

\begin{abstract}
We present $J-H-K^{\prime}$ photometry for a sample of 45 high redshift quasars found by the Sloan Digital Sky Survey. The sample was originally selected on the basis of optical colors and spans a redshift range from 3.6 to 5.03. Our photometry reflects the rest-frame SED longward of $\operatorname{Ly} \alpha$ for all redshifts. The results show that the near-IR colors of high redshift quasars are quite uniform. We have modelled the continuum shape of the quasars (from just beyond Ly $\alpha$ to $\sim 4000 \AA$ ) with a power law of the form $f_{v} \propto v^{\alpha}$, and find $\langle\alpha\rangle=-0.57$ with a scatter of 0.33 . This value is similar to what is found for lower redshift quasars over the same restframe wavelength range, and we conclude that there is hardly any evolution in the continuum properties of optically selected quasars up to redshift 5 . The spectral indices found by combining near-IR with optical photometry are in general consistent but slightly flatter than what is found for the same quasars using the optical spectra and photometry alone, showing that the continuum region used to determine the spectral indices can somewhat influence the results.
\end{abstract}

Key words. galaxies: active - quasars: general - infrared: general - cosmology: observations

\section{Introduction}

The last few years have seen a dramatic increase in the number of high redshift optically selected quasars by several surveys (e.g. Warren et al. 1994; Kennefick et al. 1995). Most prominent amongst these surveys is the Sloan Digital Sky Survey (SDSS - Fukugita et al. 1996; Gunn et al. 1998; Hogg et al. 2001; York et al. 2000; Smith et al. 2002; Stoughton et al. 2002; Pier et al. 2003; Richards et al. 2002), which has amongst its scientific aims, the construction of the largest sample of quasars ever, with more than $10^{5}$ objects spanning a large range of redshift and luminosities. The SDSS has discovered an unprecedented number of new high redshift quasars, including more than 200 new quasars at $z \geq 4$ (e.g. Fan et al. 2001a, 2000, 1999; Zheng et al. 2000; Anderson et al. 2001; Schneider et al. 2001) and the most distant quasar known to date at $z=6.4$ (Fan et al. 2003). These high redshift quasars have been efficiently selected by their distinctive position in color-color diagrams, with characteristic colors due to the main features of the quasar spectra, viz., the power law continuum, the strong Ly $\alpha$ emission line, the Ly $\alpha$ forest absorption and Lyman limit, all of which move quasars away from the stellar color locus.

Send offprint requests to: L. Pentericci, e-mail: laura@mpia.de
In this paper we aim to determine the continuum properties of high redshift quasars at optical restframe wavelengths. To do this we have obtained $J-H-K^{\prime}$ photometry of a large color-selected sample of high redshift quasars found by SDSS. A good knowledge of the quasar continuum shape at nearUV/optical restframe wavelengths is important for several reasons. Over the near-UV/optical wavelength range the shape of the continuum is usually approximated with a single power law of the form $f(v) \propto v^{\alpha}$. The mean value of $\alpha$, the dispersion of this value, and even the validity of the power law parameterization as well as the evolution of such parameters with redshift are still under debate (e.g. Vanden Berk et al. 2001 and references therein). The continuum slopes of quasars are blue, with a mean canonical spectral index of $\alpha=-0.7$ (e.g. Richstone \& Schmidt 1980), but several results of the past few years point to flatter continua (e.g. Francis 1996; Natali et al. 1998; Vanden Berk et al. 2001), and indicate a different slope at different restframe wavelength ranges. On the other hand the most recent results of Fan et al. (2001a) and Schneider et al. (2001), who find steeper average indices (respectively $\alpha=-0.8$ and -0.9$)$ for very high redshift quasars, seem to point to an evolution of the continuum properties of quasars with redshift. However, as discussed in Schneider et al. (2001), 
for high redshift quasars, determination of the continuum slope from optical data alone $\left(\lambda_{\mathrm{obs}}<1 \mu\right)$ must rely on a small restframe wavelength region with $\lambda_{\text {rest }}<1800-1900 \AA$. For example in objects at $z \sim 4$, the restframe $V$-band emission is shifted to $K$-band. Therefore near-IR data are essential to unambiguously determine the continuum properties of high redshift quasars in a way comparable to low-redshift objects, so as to get an unbiased measure of evolution.

A knowledge of the optical continuum shape and its possible redshift evolution, is not only important to understand the quasars itself but also for selecting objects at even higher redshifts. Optical colors alone begin to be less efficient for selection purposes at $z \sim 5$, since the quasars evolutionary track crosses the locus of very low-mass, late-type stars. Already, the highest redshift quasars discovered to date have been selected by adding $J$-band photometry to the SDSS colors, to distinguish quasar candidates from stars (see in particular Zheng et al. 2000 and Fan et al. 2001a).

In addition, determining the flux decrement in the Ly $\alpha$ forest needs an estimate of the continuum at $\lambda<\lambda_{\mathrm{Ly} \alpha}$, which is usually an extrapolation from $\lambda>1 \mu \mathrm{m}$. If this is done by using the classical $\alpha=-0.7$ index it can lead to uncertainties of 5-10\% in the computation of the continuum decrement. Also for high redshift quasars the observed optical fluxes must be extrapolated to obtain the optical luminosity $M_{B}$, e.g. to determine the luminosity function. Any change of $\alpha$ with redshift could substantially influence the inferred luminosity. Only with near-IR photometry can $M_{B}$ be determined directly. Lastly, for the SDSS quasar search, a knowledge of the continuum slope is essential for modeling the SDSS quasar selection function which is then used to derive the luminosity function (Fan et al. 2001b).

\section{Sample selection}

High-redshift $(z>3.6)$ quasar candidates were selected using color cuts that separate them from the stellar locus (e.g. Fan et al. 2000, 2001a). For the color-selected statistical sample presented in Sect. 4, the color criteria were applied after correcting for Galactic extinction using the reddening map of Schlegel et al. (1998). The criteria are as follows:

1. gri candidates, selected principally from the $g^{*}-r^{*}, r^{*}-i^{*}$ diagram:
(a) $i^{*}<20.05$
(b) $u^{*}-g^{*}>2.00$ or $u^{*}>21.00$
(c) $g^{*}-r^{*}>1.00$
(d) $r^{*}-i^{*}<0.42\left(g^{*}-r^{*}\right)-0.31$ or $g^{*}-r^{*}>2.30$
(e) $i^{*}-z^{*}<0.25$.

2. riz candidates, selected principally from the $r^{*}-i^{*}, i^{*}-z^{*}$ diagram:
(a) $i^{*}<20.20$
(b) $u^{*}>22.00$
(c) $g^{*}>22.60$
(d) $r^{*}-i^{*}>1.00$
(e) $i^{*}-z^{*}<0.47\left(r^{*}-i^{*}\right)-0.48$.

The intersections of those color cuts with the $g^{*}-r^{*}, r^{*}-i^{*}$ and $r^{*}-i^{*}, i^{*}-z^{*}$ diagrams are illustrated e.g. in Fan et al. (2000, 2001a).

Within these color magnitude boundaries, two subsamples were selected for observations in Spring and Fall. The first is the color selected sample in the Fall Equatorial Stripe, presented in Fan et al. (2001a), which comprises 39 objects. The second is a complete sample in the Spring Equatorial Stripe, consisting of 55 objects whose redshifts have been reported in different papers (Fan et al. 2000; Schneider et al. 2001; Anderson et al. 2001; Fan et al. 2003). Since the two samples have been selected with the same optical color criteria they can be merged together and form a large color selected sample, spanning a redshift range from 3.60 to 5.03.

The completeness of the Fall sample has been extensively discussed in Fan et al. (2001a) and is around 80\%, depending slightly on redshift; the completeness for the Spring sample should be very similar. As we detail below, we have observed a random subsample of 45 of the 94 quasars. Therefore our sample can be viewed as a statistical sample with the above color cuts.

\section{Observations and data reduction}

Observations were carried out in service mode with the MAGIC near-IR camera on the 2.2-m telescope located at Calar Alto (Spain). MAGIC is equipped with a Rockwell $256 \times 256$ pixel NICMOS3 detector array. In the high resolution mode it provides a pixel scale of $0.64^{\prime \prime}$ pixel $^{-1}$ and a total field of view of $164^{\prime \prime} \times 164^{\prime \prime}$. The observations $\log$ is shown in Table 1. In this paper we only present data from nights with photometric or nearly photometric conditions (clear nights, with very stable conditions). A fraction of the nonphotometric time was used to obtain $J$-band snapshots of candidate high redshift quasars from the SDSS, selected as $i$-band dropouts, for which subsequent spectroscopy was carried out at other telescopes (e.g. Fan et al. 2003).

Each target was observed through the $J, H$, and $K^{\prime}$ filters. The $K^{\prime}$ band (Wainscoat \& Cowie 1992) was preferred to the $K$ band to reduce the effects of thermal emission from the sky and the telescope. Each object was observed for a total ontarget exposure time between 10 and $12 \mathrm{~min}$ in each filter. For a satisfactory background subtraction, the images were dithered on a 6 position pattern ( 4 positions for the first run), with offsets of around $30^{\prime \prime}$ between each frame. We took 6, 12 or 18 frames per object depending on the night and on the brightness of the object. At each position the total integration time was split into short sub-frames to avoid saturation (the frames were directly integrated at the telescope). In Table 1 we report all these observational parameters, namely the total integration time, the subintegration times and the number of frames at each position.

During each night, 4 or more different standard stars (UKIRT faint stars from Elias et al. 1982, complemented by those observed in the same fields by Hunt et al. 1998) spanning a range of colors were observed in each of the filters at regular intervals (at least 3 times per night). In most cases the standards span the same range of airmasses as the targets. From the fall sample a total of 29 objects (out of 39) were observed under 
Table 1. Observations log.

\begin{tabular}{cccccccc}
\hline \hline Run & Date & Seeing & Nights/phot & $t_{J}$ & $t_{H}$ & $t_{K^{\prime}}$ & No. of Obj. \\
$(1)$ & $(2)$ & $(3)$ & $(4)$ & $(5)$ & $(6)$ & $(7)$ & $(8)$ \\
\hline 1 & Nov. 1999 & 1 & $1 / 1$ & $10 \mathrm{~m}(10 \mathrm{~s} \times 3)$ & $10 \mathrm{~m}(10 \mathrm{~s} \times 3)$ & $10 \mathrm{~m}(6 \mathrm{~s} \times 5)$ & 8 \\
2 & Aug. 2000 & $0.9-1.1$ & $7 / 3$ & $12 \mathrm{~m}(10 \mathrm{~s} \times 6)$ & $12 \mathrm{~m}(10 \mathrm{~s} \times 6)$ & $12 \mathrm{~m}(6 \mathrm{~s} \times 5)$ & 19 \\
3 & Feb. 2001 & $1 \leq$ & $4 / 2$ & $12 \mathrm{~m}(15 \mathrm{~s} \times 8)$ & $12 \mathrm{~m}(15 \mathrm{~s} \times 8)$ & $12 \mathrm{~m}(5 \mathrm{~s} \times 12)$ & 17 \\
4 & Mar. 2001 & & $6 / 0$ & $12 \mathrm{~m}(10 \mathrm{~s} \times 6)$ & $12 \mathrm{~m}(10 \mathrm{~s} \times 6)$ & $12 \mathrm{~m}(10 \mathrm{~s} \times 6)$ & 0 \\
5 & Jun. 2001 & $1.4-1.5$ & $2 / 1$ & $12 \mathrm{~m}(10 \mathrm{~s} \times 6)$ & $12 \mathrm{~m}(10 \mathrm{~s} \times 6)$ & $12 \mathrm{~m}(5 \mathrm{~s} \times 12)$ & 6 \\
6 & Nov. 2001 & $1-1.5$ & $3 / 2$ & $12 \mathrm{~m}(10 \mathrm{~s} \times 6)$ & $12 \mathrm{~m}(10 \mathrm{~s} \times 6)$ & $12 \mathrm{~m}(5 \mathrm{~s} \times 12)$ & 12 \\
7 & Feb. 2002 & $1.3-1.5$ & $1 / 1$ & $12 \mathrm{~m}(10 \mathrm{~s} \times 6)$ & $12 \mathrm{~m}(10 \mathrm{~s} \times 6)$ & $12 \mathrm{~m}(5 \mathrm{~s} \times 12)$ & 3 \\
\hline
\end{tabular}

(1) Run identification; (2) observation date; (3) average seeing (FWHM) during the night; (4) total number of nights/total number of photometric-good nights; (5) total integration time in $J$-band with sub-integration time and number of frames at each position; (6) the same for $H$-band; (7) the same for $K^{\prime}$-band; (9) total number of objects observed (including repetition and objects that are not in the present sample).

photometric or nearly photometric conditions. From the spring sample a total of 16 (out of 55) objects were observed. The selection of the objects was only determined by chance and observability so it is unbiased with respect to quasar properties. Therefore a total of 45 objects form a statistically well defined sample with complete near-IR photometry.

Note that the $J, H$ and $K^{\prime}$ data for a given object were taken on a single night, while the near-IR data were obtained one to two years after the optical fluxes were measured from SDSS, so we cannot exclude the possibility that variability could influence some of the results, such as the determination of the spectral indices of the continuum slopes. For example, a variability of 10-20\% in the optical flux could change the measured slope by $\sim 0.2$.

Data reduction was performed with $\mathrm{IRAF}^{1}$. The skybackground was subtracted using a composite sky frame made for each image from the previous and following images. Typically five neighboring frames were used to create a sky image, although in a few cases when the sky was changing more rapidly we used only three frames. Dome flats were obtained by taking exposures with dome lamps on and off and subtracting one from the other; these were applied after sky subtraction (an alternative flat field was also created by averaging a large number of sky-frames, masking out the brightest stars). The background subtracted, flatfielded images were shifted to a common reference, with shifts derived from centering the position of as many point sources as were visible in the frames. In most cases at least four or more such reference stars were present, but in a few objects only one reference could be used. The registered images were then co-added using the IRAF/avsigclip rejection algorithm. With the large number of frames for each target (typically 12), it was easy to perform cosmic ray rejection.

Photometric calibration was done using at least five standard stars to derive the zero point of the three bands. During each good night the scatter in the photometric zero point was less than 0.05 (slightly higher for $J$ band), without color correction so all the standard stars in a given band were simply averaged to give the final calibration. The photometry is accurate

${ }^{1}$ IRAF is distributed by the National Optical Astronomy Observatory, which is operated by the AURA, Inc under cooperative agreement with the NSF.
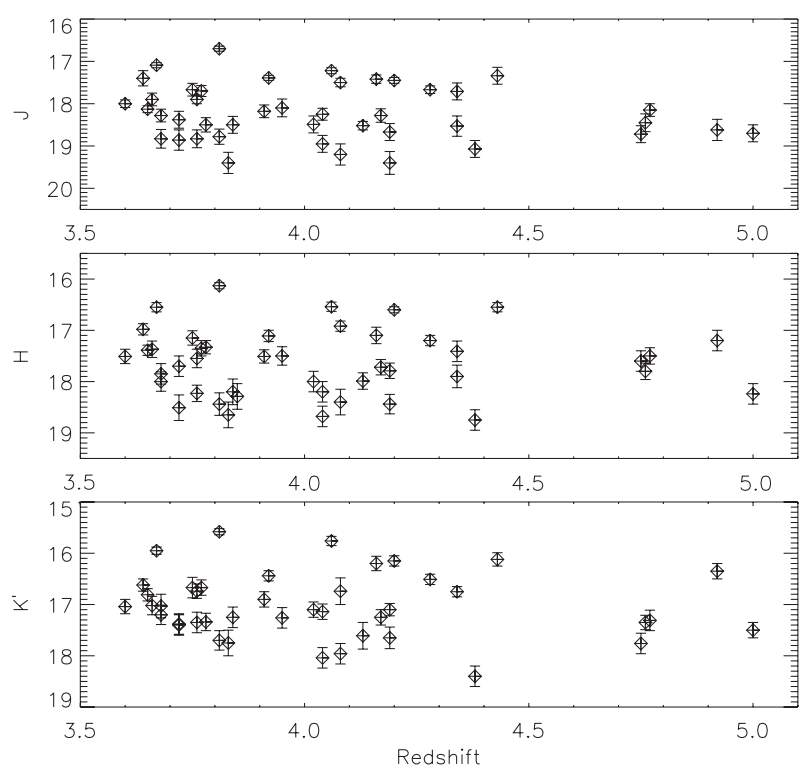

Fig. 1. The $J, H$ and $K^{\prime}$ magnitude of the quasars plotted as a function of redshift, with photometric uncertainties indicated by the errorbars.

to 0.1 magnitudes for the bright part of the sample. Magnitudes were derived inside circular apertures with radius of $4^{\prime \prime}$. The $K^{\prime}$ filter, as opposed to the standard $K$ filter, does not have many published values for standard star photometry, but for some stars interpolated $K^{\prime}$ magnitudes were available from the web page developed by Dave Thompson ${ }^{2}$. For those stars which did not have $K^{\prime}$ magnitudes available we simply used the $K$ band magnitude from Hunt et al. (1998).

\section{Results and discussion}

\subsection{Colors}

The resulting $J, H$ and $K^{\prime}$ magnitudes with their corresponding errors are presented in Table 2. The photometric errors are dominated by the sky noise. In Fig. 1 we show the distribution of $J, H$ and $K^{\prime}$ magnitudes with redshift for all quasars in the sample. In Fig. 2 we show their colors $\left(z-J, J-H\right.$ and $\left.J-K^{\prime}\right)$

\footnotetext{
2 http://www.astro.caltech.edu/mirror/keck/ realpublic/inst/nirc/UKIRTstds.html
} 


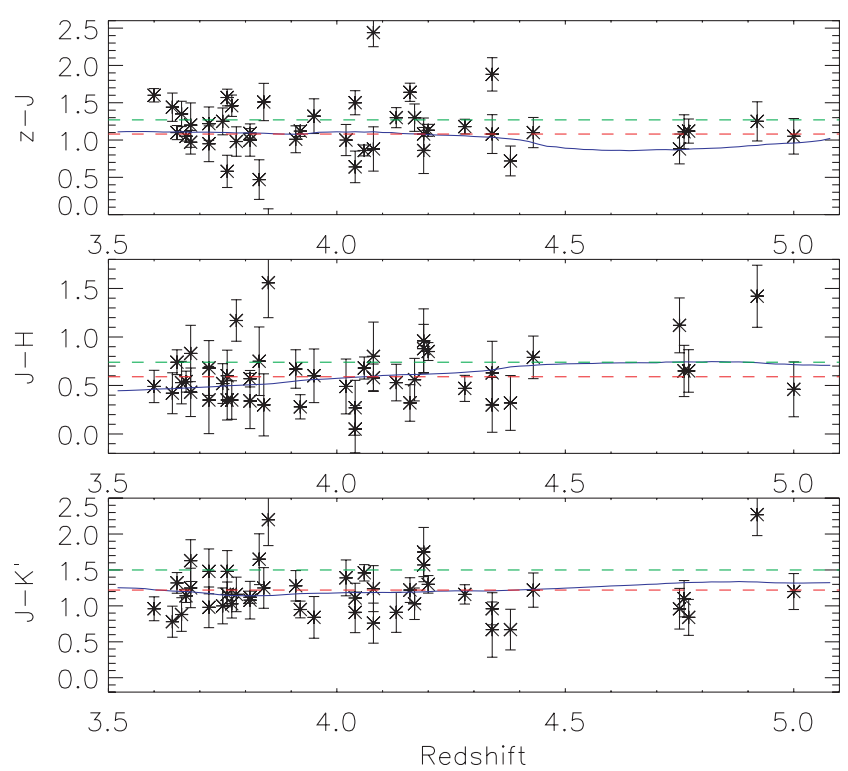

Fig. 2. The near-IR colors of the sample quasars plotted as a function of redshift. The dashed lines are the color expected from an object with a power law continuum of slope $\alpha=-0.5$ (below) and $\alpha=-1.0$ (above), while the solid lines are the colors obtained by redshifting the composite SDSS quasar spectrum (Vanden Berk et al. 2001).

again plotted as a function of redshift. Note that the $z$ band values are from SDSS photometry, based on the $A B$ magnitude system, while the IR-colors are on the Johnson (Vega based) system. In Fig. 3 we present the shape of the spectral energy distributions (SED) for all objects, combining the SDSS five-band photometry (from Fan et al. 2001a, 2000; Richards et al. 2001) with our near-IR colors. The observed flux points have been shifted to the restframe wavelength and normalized to have the same $i$-band magnitude (the $i$-band flux being of higher $S / N$ than the $z$ band). For these plots we have consistently converted the near-IR photometry into $A B$ magnitudes using the following zero points: $J_{A B}=J+0.89, H_{A B}=H+1.38$ and $K_{A B}^{\prime}=K^{\prime}+1.84$. These offsets were derived assuming that the flux density from Vega is constant within any filter and equal to 1620,1020 and $688 \mathrm{Jy}$ : the first two values are reported on the UKIRT homepage ${ }^{3}$, while for the $K^{\prime}$ filter we used an extrapolation from a measurement available in the MAGIC homepage . $^{2}$

In Fig. 2 we have also plotted as dashed lines the colors expected for a quasar if the continuum were a perfect power law with a spectral index $\alpha=-0.5$ and $\alpha=-1.0$ respectively. From the continuum only, the colors expected would be $z-J=1.08$, $J-H=0.69$ and $J-K^{\prime}=1.22$ for $\alpha=-0.5$. The solid lines in each panel represent the colors produced by shifting the composite SDSS quasar spectrum by Vanden Berk et al. (2001) to the different redshifts. This spectrum is best represented by a slightly flatter power law continuum $(\alpha=-0.46)$ and of course contains the contribution of all emission lines and features. The two lines deviate substantially from each other

\footnotetext{
${ }^{3}$ http://www. jach.hawaii.edu/JACpublic/UKIRT/ astronomy/conver.html

${ }^{4}$ http://www. caha.es/CAHA/Instruments/IRCAM/MAGIC/
}

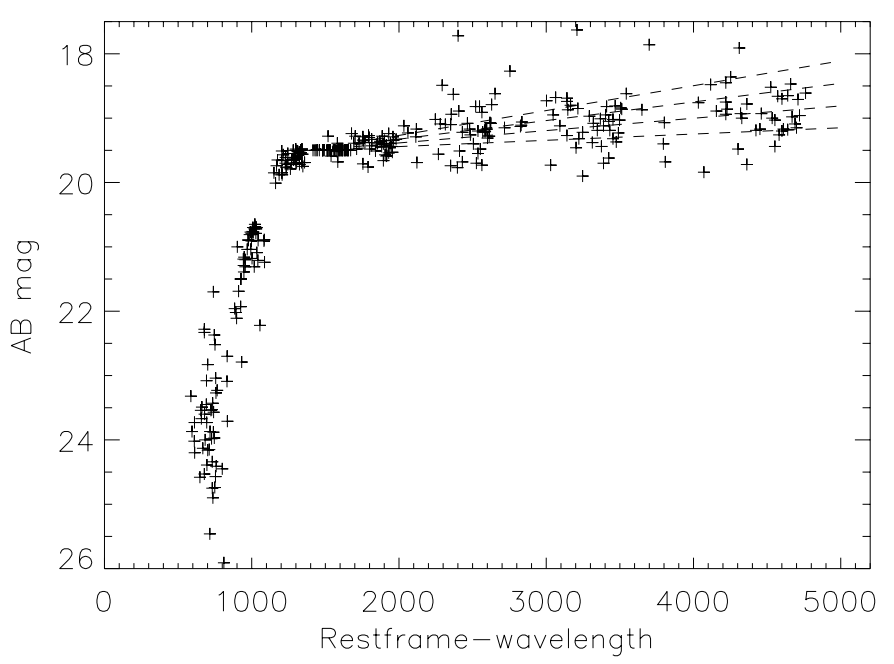

Fig. 3. The spectral energy distribution of all quasars: all near-IR magnitudes have been transformed into the $A B$ system (see text for details). The observed values have been shifted to the restframe of each object and normalized to have the same $i$-band magnitude. The lines indicate power laws with spectral indices from -0.25 to -1.0 (from the lower to the upper line). The object with colors much brighter than the other is $2256+0047$, one of those whose continuum is not well represented by a power-law. It could be also a variable quasar.

only in the $z-J$ color for objects at redshift $>4.5$, due to the contribution of CIV entering the $z$-band, and in the $J-H$ color for redshift less than $\sim 4$, due to the contribution of the MgII and the FeII complex in $J$ band (Richards et al. 2001; Barkhouse \& Hall 2001).

From the plots it is clear that the individual quasars show scatter in their colors around the predicted mean color lines. The scatter is far in excess of the measurement errors. Such scatter is produced by several factors, including the intrinsic differences of the spectral properties of quasars, which can have bluer or redder continua and the different relative strength of the emission lines in different objects. Note that in Fig. 2, the object at $z=4.92$ with colors deviating substantially from the expected ones, is a BAL quasar SDSSJ 160501.21-011220.6 (Fan et al. 2000), which shows quite unusual optical colors (see discussion in Hall et al. 2002). Apart from this object and SDSS $\mathrm{J} 103432.72-002702.6$, which is a mini BAL, there are no other BAL quasars in the sample.

There are only few near-IR measurements available in the literature for quasars at a similar redshift (e.g. Rodriguez Espinosa et al. 1988; Bechtold et al. 1994; Francis 1996; Zheng et al. 2000), all indicating colors similar to what we find.

Note that Zheng et al. (2000) used $z-J<1.5$ and $J-K<1.8$ as additional constraints to select candidate high redshift quasars, whereas Fan et al. (2001b) used only $z-J<1.5$. We see that actually all except 3 of the objects satisfy the first constraint, and only one does not satisfy the second criterion. Indeed all L and M dwarfs stars found by combined SDSS plus 2MASS photometry have $z-K>2$ (Finlator et al. 2000; Leggett et al. 2002) so their colors are quite different from those of quasars. These results confirm that the addition of one single near-IR band information to the SDSS 
Table 2. Near-IR multiband photometry of high redshift SDSS quasars.

\begin{tabular}{|c|c|c|c|c|c|c|c|}
\hline SDSS name & $J$ & $H$ & $K^{\prime}$ & $z$ & Ref. & Run & $\alpha$ \\
\hline SDSS J001950.06-004040.9 & $17.71 \pm 0.2$ & $17.41 \pm 0.15$ & $16.75 \pm 0.10$ & 4.34 & F01 & 2 & -0.79 \\
\hline SDSS J003525.29+004002.8 & - & $17.70 \pm 0.20$ & $17.72 \pm 0.15$ & 4.75 & & 1 & -0.34 \\
\hline SDSS J005922.65+000301.4 & $17.42 \pm 0.10$ & $17.10 \pm 0.16$ & $16.20 \pm 0.14$ & 4.16 & F01 & 2 & -0.94 \\
\hline SDSS J010619.25+004823.4 & $17.34 \pm 0.08$ & $16.55 \pm 0.09$ & $16.12 \pm 0.13$ & 4.43 & F99 & 2 & -0.75 \\
\hline SDSS J012019.9 & $19.20 \pm 0.35$ & $18.30 \pm 0.30$ & $17.96 \pm 0.30$ & 4.08 & F01 & 2 & -0.02 \\
\hline SDSS J012403.78+004432.7 & $16.70 \pm 0.06$ & $16.13 \pm 0.06$ & $15.58 \pm 0.06$ & 3.81 & F99 & 7 & -0.48 \\
\hline SDSS J012650.77+011611.8 & $17.90 \pm 0.15$ & $17.37 \pm 0.16$ & $17.02 \pm 0.15$ & 3.66 & F99 & 6 & -0.47 \\
\hline SDSS J012700.69-004559.1 & $17.22 \pm 0.07$ & $16.54 \pm 0.09$ & $15.76 \pm 0.09$ & 4.06 & F01 & 2 & -0.45 \\
\hline SDSS J013108.19+005248.2 & $19.40 \pm 0.27$ & $18.44 \pm 0.19$ & $17.65 \pm 0.20$ & 4.19 & F01 & 2 & -0.20 \\
\hline SDSS J015048.83+004126.2 & $17.09 \pm 0.06$ & $16.55 \pm 0.09$ & $15.95 \pm 0.07$ & 3.67 & F99 & 6 & -0.39 \\
\hline SDSS J015339.6 & $17.45 \pm 0.07$ & $16.60 \pm 0.06$ & $16.15 \pm 0.10$ & 4.20 & F99 & 2 & -0.68 \\
\hline SDSS J020731.6 & $18.90 \pm 0.20$ & $18.34 \pm 0$ & $17.70 \pm($ & 3.85 & F01 & 6 & -0.40 \\
\hline SDSS J021043.17-001818.4 & $18.15 \pm 0.13$ & $17.43 \pm 0.13$ & $17.30 \pm 0.20$ & 4.77 & F01 & 6 & -0.26 \\
\hline SDSS J023231.4 & $18.80 \pm 0.11$ & $18.60 \pm 0$ & $17.70 \pm 0$ & 3.81 & F99 & 6 & -0.03 \\
\hline SDSS J0250 & $18.45 \pm 0.21$ & $17.80 \pm 0.16$ & $17.35 \pm$ & 4.76 & F01 & 6 & -0.40 \\
\hline SDSS J02511 & $18.50 \pm 0.17$ & $3 \pm 0.13$ & $17.34 \pm$ & 3.78 & F99 & 6 & -0.20 \\
\hline SDSS J030025.2 & $18.67 \pm 0.20$ & $17.79 \pm 0.15$ & $17.10 \pm$ & 4.19 & F01 & 6 & -1.04 \\
\hline SDSS J031036.8 & $17.70 \pm 0.13$ & $17.35 \pm 0.15$ & $16.67 \pm$ & 3.77 & F01 & 7 & -0.80 \\
\hline SDSS & $8 \pm 0.12$ & 17.7 & $17.25 \pm$ & 4.17 & F01 & 3 & -0.73 \\
\hline SDSS J033829. & $18.70 \pm 0.20$ & $18.24 \pm 0$ & $17.50 \pm$ & 5.00 & F99 & 1 & -0.26 \\
\hline SDS & \pm 0.10 & 17.9 & $17.70 \pm$ & 4.13 & A01 & 3 & -0.85 \\
\hline SDSS J09163 & $18.50 \pm 0.16$ & $17.60 \pm 0$ & $17.10 \pm$ & 3.72 & F03 & 6 & -0.38 \\
\hline SDSS J0957 & $17.90 \pm 0.10$ & $17.55 \pm 0$ & $16.74 \pm$ & 3.76 & F03 & 6 & -0.67 \\
\hline SDSS & $18.13 \pm 0.08$ & 17.3 & $16.81 \pm$ & 3.65 & F03 & 3 & -0.67 \\
\hline SDS & $9.50 \pm 0.30$ & 18.5 & $17.77 \pm$ & 3.83 & S01 & 3 & -0.50 \\
\hline SDSS J103432.7 & $18.80 \pm 0.20$ & $18.43 \pm 0$ & $18.30 \pm$ & 4.38 & F03 & 3 & 0.01 \\
\hline SDSS J105602.3 & $18.45 \pm 0.15$ & $18.00 \pm 0.30$ & $17.08 \pm 0$ & 4.00 & F03 & 1 & -0.61 \\
\hline SDSS J111224.18+004630.4 & $18.94 \pm 0.20$ & $18.60 \pm 0.25$ & 17.9 & 4.04 & A01 & 3 & 0.09 \\
\hline SDSS J111246.29+004957.5 & $17.39 \pm 0.06$ & $17.11 \pm 0.11$ & $16.44 \pm 0$ & 3.92 & F00 & 3 & -0.30 \\
\hline SDSS J134723.09+002158.9 & $17.67 \pm 0.09$ & $17.20 \pm 0.10$ & $16.51 \pm 0.10$ & 4.28 & A01 & 3 & -0.51 \\
\hline SDSS J135134.46-003652.2 & $18.25 \pm 0.10$ & $18.25 \pm 0.20$ & $17.14 \pm 0.15$ & 4.04 & A01 & 3 & -0.76 \\
\hline SDSS J1358 & $18.18 \pm 0.10$ & $17.51 \pm 0.10$ & $16.90 \pm 0$ & 3.91 & F03 & 5 & -0.51 \\
\hline SDSS J140754.54+001312.1 & $18.86 \pm 0.20$ & $18.50 \pm 0.30$ & $17.30 \pm 0.20$ & 3.72 & F03 & 3 & -0.49 \\
\hline SDSS J14232 & $18.83 \pm 0.17$ & $18.20 \pm 0.15$ & $17.40 \pm 0.20$ & 3.76 & F00 & 3 & -0.08 \\
\hline SDSS J142647.8 & $18.28 \pm 0.10$ & $17.80 \pm 0.15$ & $17.03 \pm 0$ & 3.68 & F00 & 5 & -0.41 \\
\hline SDSS J160501.21-011220.6 & $18.30 \pm 0.26$ & $17.20 \pm 0.10$ & $16.40 \pm 0.10$ & 4.92 & F00 & 2 & -1.72 \\
\hline SDSS J162500.04-000128.5 & $18.00 \pm 0.09$ & $17.51 \pm 0.14$ & $17.04 \pm 0.14$ & 3.60 & F03 & 5 & -1.03 \\
\hline SDSS J225624.35+004720.2 & $17.48 \pm 0.10$ & $16.90 \pm 0.10$ & $16.74 \pm 0$ & 4.08 & F01 & 2 & -1.77 \\
\hline SDSS J225759.67+001645.7 & $17.67 \pm 0.15$ & $17.15 \pm 0.14$ & $16.60 \pm 0.15$ & 3.75 & F99 & 2 & -0.34 \\
\hline SDSS J230323.77+001615.2 & $18.83 \pm 0.20$ & $18.25 \pm 0.15$ & $17.20 \pm 0.18$ & 3.68 & F01 & 5 & -0.80 \\
\hline SDSS J230639.65+010855.2 & $17.40 \pm 0.18$ & $16.98 \pm 0.11$ & $16.62 \pm 0.12$ & 3.64 & F01 & 6 & -0.67 \\
\hline SDSS J230952.29-003138.9 & $18.10 \pm 0.21$ & $17.50 \pm 0.14$ & $17.26 \pm 0.20$ & 3.95 & F99 & 2 & -0.47 \\
\hline SDSS J232208.22-005235.2 & $18.60 \pm 0.20$ & $18.14 \pm 0.20$ & $16.80 \pm 0.10$ & 3.84 & F01 & 6 & -0.83 \\
\hline SDSS J235718.35+004350.4 & $18.53 \pm 0.20$ & $17.97 \pm 0.18$ & $17.86 \pm 0.30$ & 4.34 & F99 & 2 & -0.86 \\
\hline
\end{tabular}

References for redshift: F99: Fan et al. (1999); F01: Fan et al. (2001a); F03: Fan et al. (2003, in preparation); A01: Anderson et al. (2001); S01: Schneider et al. (2001).

photometry can greatly improve the efficiency of finding high redshift quasars.

\subsection{Continuum slopes}

The continuum slopes were computed using all SDSS bands that fall fully redward of the Ly $\alpha$ line (i.e. excluding the band that contains $\operatorname{Ly} \alpha$ ) in combination with the three near-IR bands.
As already noted in the previous section, contamination by line emission can be important in some bands. The most important lines that are present in the quasar spectra at the restframe wavelengths sampled by our observations are the CIV $\left(\lambda_{\text {rest }}=1549 \AA\right)$ and CIII] $\left(\lambda_{\text {rest }}=1909 \AA\right)$ lines for the optical photometry ( $i$ and $z$-band); the $\operatorname{MgII}$ line $\left(\lambda_{\text {rest }}=2798 \AA\right.$ ) which falls in the $J$-band for objects with $z<4.0$ and in the $H$-band for objects with $z>4.36$; finally $\mathrm{H} \beta\left(\lambda_{\text {rest }}=4861 \AA\right)$ 

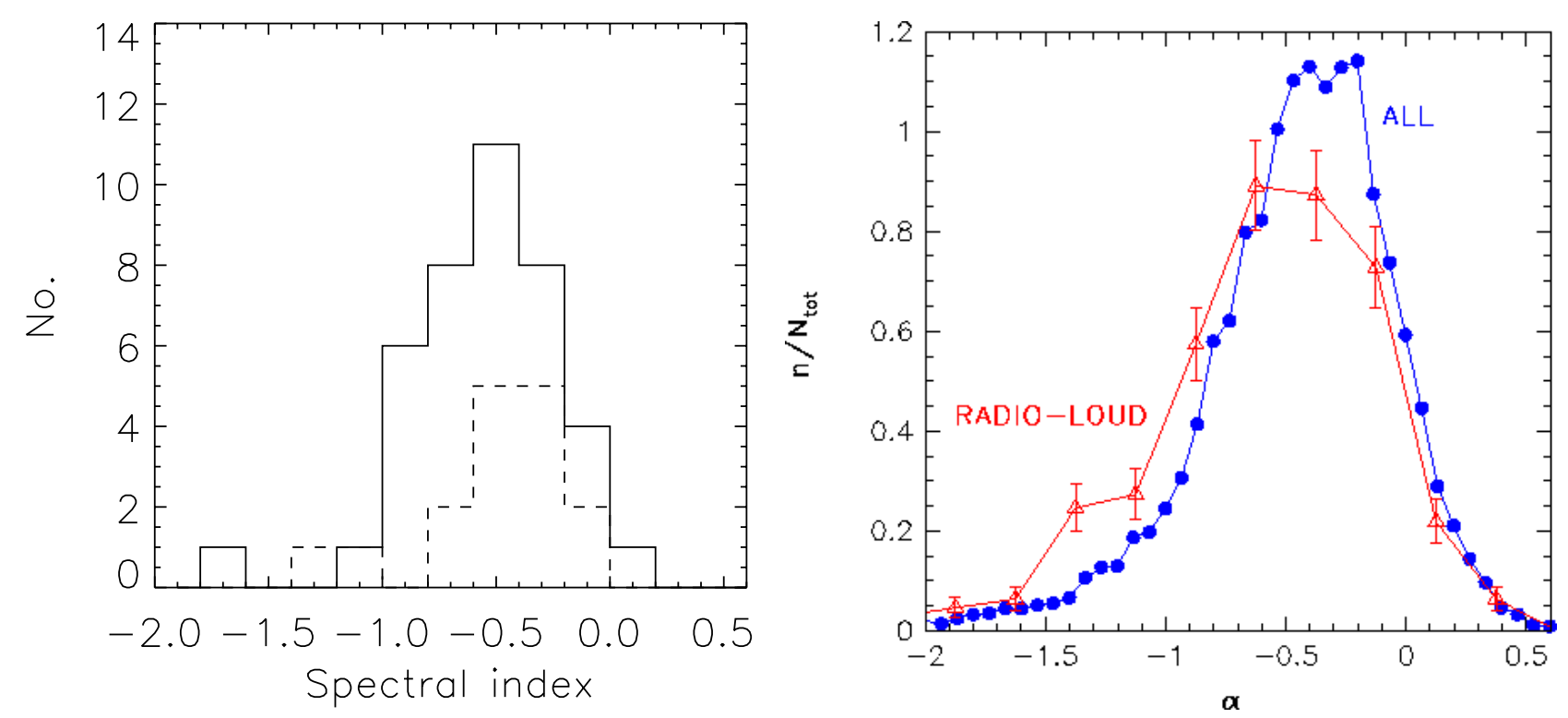

Fig. 4. Left: the distribution of the quasars continuum power-law index $\alpha$ from the combined fall and spring samples. The dotted line represents the distribution of spectral indices from a sample of quasars at redshift between 2 and 3.5 from Francis (1996). Right: the distribution of spectral indices derived by Ivezić et al. (2002) for about 6800 SDSS quasars with magnitude brighter than $i=19$. The $\alpha$ distribution for a subsample of 440 radio-loud quasars, indicated by the triangles, is skewed towards more negative values.

which contaminates the $K^{\prime}$ band for objects with $z<3.73$. Many other lines also fall in these regions but their contributions to broad band fluxes are mostly negligible. A special case is the very broad feature that appears between $2200-4000 \AA$, known as the $3000 \AA$ bump, which consists of blends of FeII line emission and Balmer continuum emission (e.g. Wills et al. 1985; Richards et al. 2002). No attempt was made to correct for these features since their contribution is hard to disentangle. However, we have seen from the color distribution in the previous section that this contribution cannot be very large, or at least it cancels out, otherwise we would observe larger departures from the values expected from a simple power law continuum. Furthermore we are using a quite large wavelength baseline (from 7000 to $22000 \AA$ ) which means that these errors would not have a large effect on the derived continuum slope. For example an error of 25 per cent in the flux of one of the bands would introduce an error of $\leq 0.1$ in $\alpha$ (in the sense that if the $25 \%$ was due to the flux contributed by the FeII bump, the true $\alpha$ would be flatter than what we measure).

For a large fraction of the objects in the sample the strength of the CIV line is known, having been measured from the low resolution discovery spectra (Fan et al. 1999, 2001a, 2003 in preparation; Anderson et al. 2001; Schneider et al. 2001). The strength of the other lines was not known so we used the average equivalent width derived from the combined spectra of SDSS quasars by Vanden Berk et al. (2001). In particular we used $E W=23.78 \AA$ for CIV (when the real value was not available), $E W=21.19 \AA$ for CIII], $E W=34.95 \AA$ for $\mathrm{MgII}$ and $E W=46.21 \AA$ for $\mathrm{H} \beta$ (these are all restframe values). Note that in most cases the contribution of line emission flux to the broad band flux is less than $0.1 \mathrm{mag}$, so it is comparable to or less than the photometric error.

We subtracted the line contribution from the relevant broad band values and then converted magnitudes into flux.
The corrected continuum fluxes were then modeled with a power law of the form $f_{v} \propto v^{\alpha}$ and the best fitting spectral index $\alpha$ was derived by least squares minimization. The fit by a power law was considered acceptable when the probability that a value of chi-square as poor as the value found should occur by chance was larger than 0.05 . For 5 objects in the sample (0019-0040, 0035+0040, 1625-0001, 2256+0047 and $2306+0108)$ this requirement was not satisfied, so we report the values we have obtained in Table 2 , but we do not include them in the discussion.

In Fig. 4 we present the distribution of $\alpha$ from the combined Fall and Spring sample. The distribution is essentially confined between 0.2 and -1.1 (with one object at -1.8 ), with an average spectral index of $\alpha=-0.57$ and $1 \sigma$ dispersion of 0.33 . The mean was weighted by the errors on the individual $\alpha$ measurements. The median value is $\alpha=-0.51$. The distribution is not perfectly symmetric, but slightly skewed towards steeper indices. In Fig. 4 (right panel) we also show for comparison the distribution of spectral indices derived by Ivezić et al. (2002) for about 6800 SDSS quasars with magnitude brighter than $i=19$, spanning a large range of redshifts (see also discussion in Richards et al. 2002). The two distributions appear similar. The values found for our sample are consistent with the average power law index derived from the composite SDSS quasar spectra, spanning a redshift range $0.04<z<4.79$ (Vanden Berk et al. 2001) which is $\alpha=-0.47$. This index is valid for a large spectral range, $1216 \AA \leq \lambda \leq 5000 \AA$ restframe: at longer wavelengths, the slope changes to $\alpha=-1.58$. A similar result was obtained by Francis (1996) who derived $\alpha$ using photometric estimates, including near-IR photometry, for a sample of quasars spanning a redshift range 0.33 to 3.67 and with a median redshift of 2. In Fig. 4 we plot the spectral indices derived by Francis (1996) for all the objects with redshift above 2: for these objects the average spectral index is 


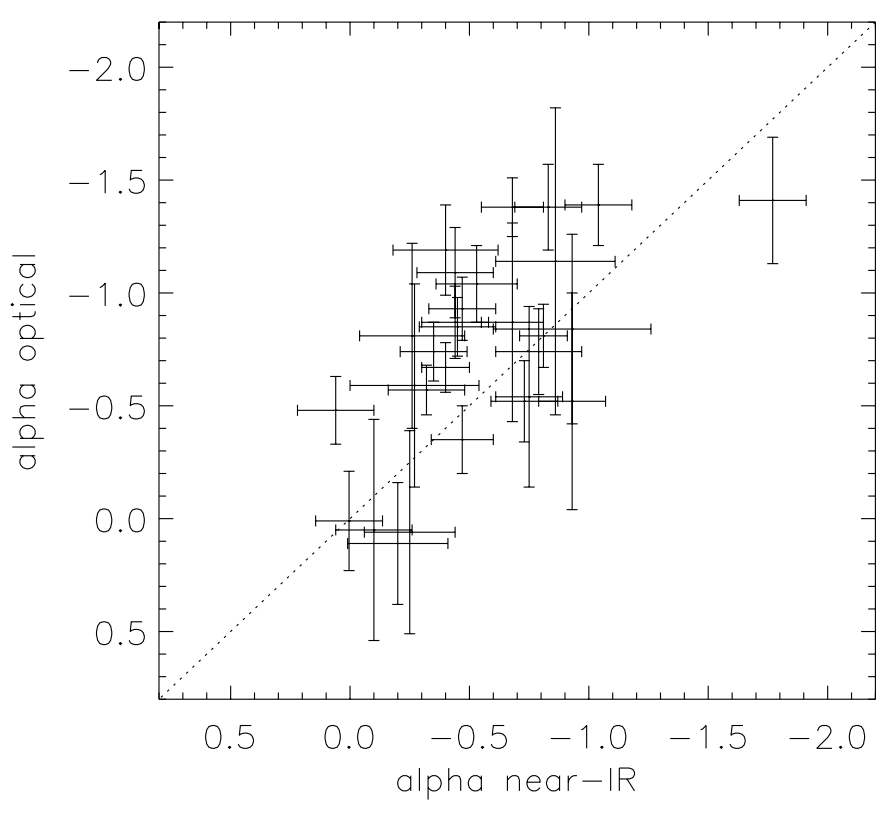

Fig. 5. The spectral index of the continuum emission: on the $y$ axis is the value derived by Fan et al. (2001) from the low resolution spectra, on the $x$-axis the value derived from optical and near-IR photometry. The error bars represent the $1 \sigma$ uncertainties.

$\alpha=-0.49$ (median -0.43 ). Natali et al. (1998) find a similar result for a sample of bright quasars with redshift up to 2.5, with $\alpha=-0.65$. However this slope is based on the continuum shortward of the $3000 \AA$ bump, with a much flatter index at $4000 \AA$ Å. One discrepant result comes from Kuhn et al. (2001), who derived the slopes of a sample of bright quasars with redshift around 3, using both photometry and spectroscopy. They find slightly flatter average spectra, with $\alpha=-0.34$ (median -0.29); however we note that their spectral slopes for the $z \sim 0$ comparison sample of quasars are also flatter than what was found in other studies.

In Fig. 5 we plot the derived values for the Fall sample, and compare them to the spectral indices derived for the same quasars in Fan et al. (2001a) from the low resolution spectra and optical photometry. The error bars plotted in the figure indicate the $1 \sigma$ error. Basically all spectral indices are consistent with those derived from optical data within $3 \sigma$. However the indices derived from the combined near-IR and optical broad band photometry tend to be flatter (bluer) than those derived from the optical data alone and the dispersion of $\alpha$ values around the mean is also somewhat smaller. The average spectral index derived from the photometry for the $29 \mathrm{ob}-$ jects of the fall sample alone is $\alpha=-0.59 \pm 0.34$ with a median value of -0.47 , whereas for the compete fall sample (all 39 quasars) Fan et al. (2001a) derived an average value of $\alpha=-0.88 \pm 0.36$ (note however that they applied weights that included the error measurement as well as the detection probability function). Similarly Schneider et al. (2001) derived an average $\alpha=-0.93 \pm 0.31$ from spectra of high redshift SDSS quasars, some of which are included in our sample.

The apparent discrepacy probably arises because the indices are derived from different wavelength regions of the continuum emission: the indices derived by Fan et al. (2001a) and by Schneider et al. (2001) are from the continuum immediately blueward of the Ly $\alpha$ up to $9000 \AA$ (or less) observed emission, and thus include only a few hundred $\AA$ in the restframe (1250 to $1600-1800 \AA$ depending on the redshift). Furthermore, part of the Fe complex in the 1500-2000 A region could make the $i$ and $z$ band brighter, thus producing a steepening of the spectral indices. On the other hand our photometry spans a much larger wavelength region and, most important, includes longer wavelengths: at redshift 3.65 (our lower redshift) the photometry cover from 1500 to $4700 \AA$ (restframe) whereas at redshift 5, our most distant object, the coverage goes from $\sim 1150 \AA$ up to $\sim 3700 \AA$. Figure 3 illustrates that the continuum immediately longward of $\operatorname{Ly} \alpha$ is somewhat redder than at longer wavelengths.

We conclude that the continuum properties of the high redshift quasars in our sample at optical restframe wavelengths are comparable to those of their lower redshift counterparts, with no significant change with epoch. We also find that the spectral slopes change somewhat depending on the wavelength regions used to measure them, as already indicated by the results of Vanden Berk et al. (2001) and Natali et al. (1998).

\section{Summary}

We have presented near-IR photomety $\left(J, H, K^{\prime}\right)$ of a sample of 45 color-selected SDSS quasars at $3.6<z<5$. We have determined the slopes of the optical continuum at restframe wavelengths $1200 \AA<\lambda_{\text {rest }}<5000 \AA$, and we find an average spectral slope $\alpha=-0.57$ and a $1 \sigma$ dispersion of 0.33 . This value is consistent with those found by many authors for lower redshft quasars at similar restframe wavelengths, so we conclude that there is no evidence for a redshift evolution of the quasar continuum properties, as it was previously suggested. By comparing our slopes with those determined by Fan et al. (2001) for the same objects, using the optical spectroscopy and photometry alone, we can see that the addition of near-IR data yields a somewhat bluer continuum and more robust evaluation. This implyes that the determination of the spectral slope changes somewhat depending on the wavelength used to measure them, as already indicated by previous results.

Acknowledgements. This paper is based on observations in the framework of the "Calar Alto Key Project for SDSS Followup Observations" (Grebel 2001) obtained at the German-Spanish Astronomical Centre, Calar Alto Observatory, operated by the Max Planck Institute for Astronomy, Heidelberg jointly with the Spanish National Commission for Astronomy.

Funding for the creation and distribution of the SDSS Archive has been provided by the Alfred P. Sloan Foundation, the Participating Institutions, the National Aeronautics and Space Administration, the National Science Foundation, the U.S. Department of Energy, the Japanese Monbukagakusho, and the Max Planck Society. The SDSS Web site is http://www.sdss.org/. The SDSS is managed by the Astrophysical Research Consortium (ARC) for the Participating Institutions. The Participating Institutions are The University of Chicago, Fermilab, the Institute for Advanced Study, the Japan Participation Group, The Johns Hopkins University, Los Alamos National Laboratory, the Max-Planck-Institute for Astronomy (MPIA), the Max-Planck-Institute for Astrophys ics (MPA), New Mexico State University, University of Pittsburgh, 
Princeton University, the United States Naval Observatory, and the University of Washington. MAS acknowledges the support of NSF grant AST 00-71091

\section{References}

Anderson, S. F., Fan, X., Richards, G T., et al. 2001, AJ, 122, 503

Barkhouse, W. A., \& Hall, P. B. 2001, AJ, 121, 2843

Bechtold, J., Elvis, M., Fiore, F., et al. 1994, AJ, 108, 374

Elias, J. H., Frogel, J. A., Matthews, K., \& Neugebauer, G. 1982, AJ, 87,1029

Fan, X., Strauss, M. A., Schneider, D. P., et al. 1999, AJ, 118, 1

Fan, X., Strauss, M. A., Schneider, D. P., et al. 2000, AJ, 119, 1

Fan, X., Strauss, M. A., Richards, G. T., et al. 2001a, AJ, 121, 31

Fan, X., Strauss, M. A., Schneider, D. P., et al. 2001b, AJ, 121, 54

Fan, X., Strauss, M. A., Schneider, D. P., et al. 2003, AJ, 125, 1649

Finlator, K., Ivezic, Z., Fan, X., et al. 2000, AJ, 120, 2615

Francis, P. J. 1996, Publ. Astron. Soc. Austr., 13, 212

Fukugita, M., Ichikawa, T., Gunn, J. E., et al. 1996, AJ, 111, 1748

Grebel, E. K. 2001, Rev. Mod. Astron., 14, 223

Gunn, J. E., Carr, M., Rockosi, C., et al. 1998, AJ, 116, 3040

Hall, P. B., Anderson, S. F., Strauss, M. A., et al. 2002, ApJS, 141, 267

Hogg, D., Finkbeiner, D. P., Schlegel, D. J., \& Gunn, J. E. 2001, AJ, 122,2129

Hunt, L. K., Mannucci, F., Testi, L., et al. 1998, AJ, 115, 2594
Ivezic, Z., Menou, K., Knapp, G. R., et al. 2002, AJ, 124, 2364

Kuhn, O., Elvis, M., Bechtold, J., \& Elston, R. 2001, ApJS, 136, 225

Kennefick, J. D., de Carvalho, R. R., Djorgovski, S. G., et al. 1995, AJ, 110, 78

Leggett, S. K., Golimowski, D. A., Fan, X., et al. 2002, ApJ, 564, 452

Natali, F., Giallongo, E., Cristiani, S., \& La Franca, F. 1998, AJ, 115, 397

Pier, J., Munn, J. A., Hindsley, R. B., et al. 2003, AJ, 125, 1559

Richards, G. T., Fan, X., Schneider, D. P., et al. 2001, AJ, 121, 2308

Richards, G. T., Fan, X., Newberg, H. J., et al. 2002, AJ, 123, 2945

Richstone, D. O., \& Schmidt, M. 1980, ApJ, 235, 361

Rodriguez Espinosa, J. M., Stanga, R. M., \& Moorwood, A. F. M. 1988, A\&A, 192, 13

Schlegel, D. J., Finkbeiner, D. P., \& Davis, M. 1998, ApJ, 500, 525

Schneider, D. P., Fan, X., Strauss, M. A., et al. 2001, AJ, 121, 1232

Smith, J. A., Tucker, D. L., Kent, S., et al. 2002, AJ, 123, 2121

Stoughton, C., Lupton, R. H., Bernardi, M., et al. 2002, AJ, 123, 485

Vanden Berk, D. E., Richards, G. T., Bauer, A., et al. 2001, AJ, 122, 549

Wainscoat, R., \& Cowie, L. 1992, AJ, 103, 332

Warren, S. J., Hewett, P. C., \& Osmer, P. S. 1994, ApJ, 421, 412

Wills, B. J., Netzer, H., \& Wills, D. 1985, ApJ, 288, 94

York, D. G., Adelman, J., Anderson, J. E, Jr., et al. 2000, AJ, 120, 1579

Zheng, W., Tsevtanov, Z. I., Schneider, D. P., et al. 2000, AJ, 120, 1607 